La décentralisation de la gestion des ressources forestières au Sénégal : un processus contraint par le marché?

Laurence Boutinot

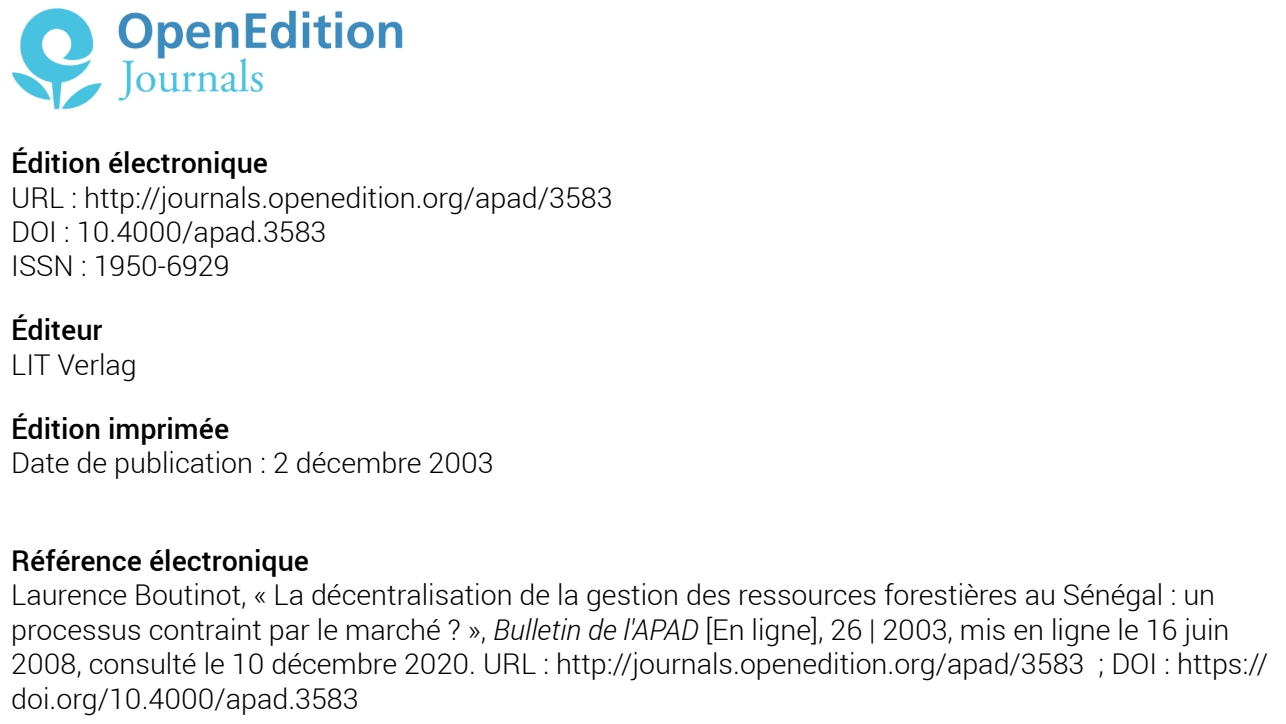

Ce document a été généré automatiquement le 10 décembre 2020.

Bulletin de l'APAD 


\title{
La décentralisation de la gestion des ressources forestières au Sénégal : un processus contraint par le marché ?
}

\author{
Laurence Boutinot
}

Introduction

1 Dans le domaine de la gestion des ressources naturelles, les situations des pays d'Afrique de l'Ouest francophone sont fort différentes tant au niveau national qu'au sein même de chaque pays. Pourtant, malgré les mises en œuvre plus ou moins linéaires des politiques de décentralisation, nous constatons un certain nombre de questions récurrentes que ces réformes ne manquent pas de soulever. Ces questions relèvent la plupart du temps des conflits d'autorité autour du contrôle des territoires et des ressources qu'ils comprennent. Les plus fréquents confrontent les services de l'État à travers notamment les institutions forestières, les élus locaux et les chefferies coutumières. Ils évoquent à la fois la place accordée aux pouvoirs coutumiers dans les dispositifs décentralisés, les stratégies d'appropriation foncière et les référents identitaires (ré)invoqués et (re)mobilisés autour des enjeux pour l'accès aux ressources ${ }^{1}$.

2 Une des particularités du Sénégal peut se lire au travers de l'importance accordée aux productions de bois et de charbon de bois dont la filière économique, contrairement aux pays voisins, est ancienne et fortement structurée.

3 L'objet de cette contribution est de rendre compte de la manière dont ces enjeux se traduisent à l'heure actuelle au Sénégal, dans les relations entre différents acteurs : les élus locaux (présidents des Communautés rurales et conseillers ruraux), les chefs de villages, les agents des Eaux et Forêts et les exploitants privés. Au milieu de ces acteurs, certains projets de développement participatifs (programmes nationaux et/ ou projets d'ONG) pour la gestion des ressources naturelles jouent un rôle particulier, voire une "fonction parapublique", qui n'est pas sans produire des effets sur ces rapports de 
force, contrariant parfois plus qu'ils ne favorisent le processus de décentralisation démocratique, pour autant que l'on regarde celui-ci comme un mouvement potentiel de redistribution des ressources économiques.

La décentralisation : un mouvement non linéaire et des contradictions Un siècle de changements rapides

4 Le Sénégal est engagé dans un processus de décentralisation qui est très ancien puisqu'il commence dès le $\mathrm{XIX}^{\mathrm{e}}$ siècle. Mais depuis la création des premières communes de plein exercice (Saint Louis et Gorée en 1872, Rufisque en 1880 et Dakar en 1887) il se présente comme un mouvement espacé et s'inscrivant dans les linéaments de différentes grandes périodes politiques. Processus concernant dans un premier temps les centres urbanisés, il s'élargit aux communes de Thiès, Kaolack, Ziguinchor, Diourbel et Louga par la loi française de 1955 à la fin de la période coloniale. Il s'amorce cependant à la même époque dans le monde rural à travers un décret de 1957 qui donne aux chefs de territoire le pouvoir de créer des communautés rurales bénéficiant de la personnalité morale et d'une indépendance financière. Ainsi, dès 1960, le pays compte une trentaine de communes de plein exercice. On observe alors un ralentissement du processus, voire un recul comme dans nombre de pays de la sous région non sans lien avec la volonté post-coloniale d'unification politique nationale. En 1972 une nouvelle réforme territoriale institue des communes à régime spécial (exécutif nommé) et des communautés rurales dotées d'un conseil élu mais d'un exécutif revenant au souspréfet. Vingt ans après, les communes à régime spécial et les pouvoirs exécutifs nommés sont supprimés.

5 En fait, le stade récent de la décentralisation s'affirme à travers la loi de 1996. Mais ce ne fut pas sans mal et il n'apparait pas étonnant que cette loi ait fait l'objet de débats parlementaires et pas moins de trois cent vingt amendements de la part des députés avant sa mouture finale. La loi sur la décentralisation de 1996 porte création de la région, de la commune et de la communauté rurale en tant que collectivités locales dotées d'un conseil élu au suffrage universel pour 5 ans. Ce conseil désigne en son sein un président de communauté rurale (ou un maire dans une commune urbaine) et un adjoint, formant le pouvoir exécutif.

6 Par la même loi, les collectivités locales sont dotées au Sénégal de compétences spécifiées et concernant des domaines importants de la gestion des affaires publiques dont la santé, l'action sociale, la jeunesse et les sports, la culture, l'éducation, la planification et l'aménagement du territoire, l'environnement et la gestion des ressources naturelles. Les élus des différents paliers de la décentralisation ont créé les associations des présidents des conseils ruraux (APCR), des Maires (AMS) et des présidents de conseils régionaux (APR).

7 Si le processus semble bien se renforcer "officiellement" depuis l'alternance politique de mars 2000, dans le même temps et durant la seule période récente de l'alternance démocratique du Président Abdoulaye Wade, nous avons assisté à des recadrages successifs du Ministère de la décentralisation, comme, du reste, dans l'ensemble du gouvernement. Ainsi, après les élections législatives de 2001, le Ministère de la décentralisation et de l'aménagement du territoire est remplacé par deux instances différentes : le Ministère délégué aux collectivités locales et celui de l'urbanisme et de l'aménagement. Descendu au rang de ministère délégué en 2002, il sera sous la tutelle du Ministère de la Défense. Le décret du 28 août 2003, nomme Macky Sall, Ministre d'État, ministre de l'intérieur, des collectivités locales, et porte parole du 
gouvernement. Enfin depuis avril 2004, les collectivités locales reviennent dans un ministère d'État, dirigé par $\mathrm{Mr}$ Aminata Tall. Par ailleurs, du processus de déconcentration censé équilibrer et accompagner celui de la décentralisation, on n'en parle guère.

Mais on ne voit jamais aussi bien les indécisions de l'État dans la mise en œuvre de la politique de décentralisation qu'à la faveur de quelques exemples empiriques de gestion locale.

9 Rappelons toutefois la difficulté d'un tel processus en regard d'une histoire coloniale qui rendait déjà difficile le projet de décentralisation. L'encadrement administratif et autoritaire des anciennes colonies préparait mal, en effet, aux nouvelles formes de relations entre l'État et ses "assujettis" (Billaz, 2003 : 29). De surcroît, la souveraineté des États aux lendemains des indépendances sur des territoires dont les frontières tracées moins d'un siècle auparavant ont pu être parfois controversées rendaient déjà les unités nationales difficiles à construire sur des mosaïques de sociétés et de cultures partageant des espaces entre lesquels les voies de communication sont toujours insuffisantes voire, par endroit, encore inexistantes.

10 Mais c'est aussi dans la rapidité du développement urbain et de la croissance démographique des cinquante dernières années, les endettements des années 1970 et les fortes demandes de services publics (logement, école, dispensaire, infrastructures routières, etc.) consécutives à ces bouleversements, que l'on mesure également la difficulté de l'exercice de la décentralisation. "Au moment où les instances internationales ont sévèrement critiqué la gouvernance de ces pays, il aurait été prudent qu'elles se rappellent qu'aucun pays occidental n'avait jamais eu à faire face à de tels défis" (Billaz, $2003: 33$ ).

11 Aujourd'hui justifiée par l'ouverture démocratique et la "bonne gouvernance" par nombre d'État africains et par les institutions internationales, la décentralisation reste étroitement liée aux obligations des politiques d'ajustement structurel et contrainte dans la logique de réforme libérale. Aussi, ne sert-elle, en définitive, que très peu les principes à partir desquels elle se justifie officiellement. Bien que les textes de lois et décrets d'application réglementant le processus de décentralisation soient, au Sénégal, déjà suffisants pour mettre en place les réformes, ces dernières concernant l'administration tiennent peu compte des processus en cours (Samb, 2004: 50) et, de fait, les pouvoirs de décisions se contredisent plus qu'ils ne se complètent au niveau local.

La gestion des ressources naturelles : un domaine de compétence sensible?

12 La gestion des ressources naturelles qui inclut autant la gestion du foncier que celle des ressources renouvelables (eau, arbre) participe de la définition d'un territoire, fondement de la souveraineté des États, et l'enjeu de leur gestion est tel qu'il n'est pas rare de constater les réticences de certains États à transférer aux collectivités locales cette compétence spécifique dans le cadre des politiques de décentralisation. La Côte d'Ivoire, la Guinée, la Mauritanie, par exemple, malgré l'ancienneté très variable de leur démarche décentralisatrice, sont restés en effet prudents dans la délégation de la gestion de leur patrimoine foncier et domanial.

13 Mais au delà de la question foncière, qui est, du reste au Sénégal désormais déléguée aux commissions domaniales des collectivités locales, les questions forestières relèvent d'enjeux particuliers. Car parmi les neuf compétences transférées par la loi aux collectivités locales sénégalaises, celle de la gestion des ressources naturelles, et 
notamment forestières, est une source de revenus importante, notamment pour les communautés rurales riveraines des massifs forestiers au sud du pays. Rappelons que la seule production de charbon de bois rapporte à l'État près de 20 milliards de francs CFA par an. Et les recettes provenant des amendes et confiscations, dont les données chiffrées exactes restent délicates à obtenir, n'en sont pas moins éloquentes et en constante évolution. Ainsi, entre 1990 et 2000 elles sont respectivement passées, d'après les sources de la DEFCCS ${ }^{2}$ de 50355565 Francs CFA à 127634785 Francs CFA. Et il n'est pas irréaliste de penser qu'il s'agit là de la part immergée des flux réels.

La gestion des ressources forestières est lourde d'un passé conflictuel entre les services de l'État et les usagers ${ }^{3}$. Les nouvelles formes de gestion locales mises en place par les forestiers eux mêmes à travers des programmes de développement participatifs ou par des ONG, contribuent sans nul doute à modifier et à améliorer durablement ces relations. Cependant, nous le verrons, ces initiatives restent en deçà d'une véritable volonté de transférer des pouvoirs et une certaine autonomie de décision aux conseils ruraux censés représenter ces usagers. Rappelons que les politiques forestières restent historiquement soucieuses de maintenir plusieurs aspects fondamentaux de leurs prérogatives : le contrôle des territoires, la fonction de protection des forêts et son corollaire qui considère les usagers comme les principaux responsables des dégradations et qui, partant, justifie les pratiques d'exclusion et de répression; et enfin le souci d'une réglementation spécifique qui prend surtout en compte les aspects économiques et commerciaux, et notamment la production de bois et de charbon de bois (Buttoud, 1995 : 41).

Avec la notion de "terres vacantes et sans maîtres" les services forestiers ont longtemps maintenu leur domination sur l'ensemble des terres boisées. Mais depuis les codes forestiers de 1993 et plus particulièrement depuis celui de 1998, la définition des forêts appartenant au domaine forestier de l'État et sous la responsabilité directe des services forestiers limite celles-ci à :

"L'ensemble des zones classées comprenant les forêts classées, les réserves sylvopastorales, les périmètres de reboisement et de restauration, les parcs nationaux, les réserves naturelles intégrales et les réserves spéciales". (art. R $2 \mathrm{~N}^{\circ}$ 98-164)

La décentralisation concède ainsi un important pouvoir de gestion aux communautés rurales sur les espaces forestiers relevant de leurs limites administratives. Mais ces communautés rurales ont bien du mal à exercer leurs prérogatives nouvelles sur ces espaces qui sont des lieux convoités par divers acteurs dont les pratiques d'appropriation sont également rendues possibles par les mêmes lois. Ainsi, les possibilités pour des acteurs privés d'amodier des espaces de forêts communautaires pour créer des espaces cynégétiques et touristiques passent par l'avis du conseil rural certes, mais aussi par le conseil régional. Ce dernier n'ayant pas de territoire à proprement parler, obtient par cette disposition, une opportunité d'accéder à des ressources naturelles et à des revenus supplémentaires. De même, la volonté politique de créer des forêts régionales, participe du même mouvement, sorte de restrictions des espaces dévolus par la même loi aux communautés rurales.

De plus, la présence des agents forestiers dans toutes les commissions régionales, ainsi que dans les commissions techniques et le rôle éminent du Ministère de l'Environnement contribuent au contrôle rapproché des processus de décision ${ }^{4}$.

La décentralisation à trois paliers nécessite déjà des financements importants en matière d'aménagement infrastructurel et technique et demande une politique 
d'aménagement soucieuse d'un découpage territorial réaliste. Or certaines régions du pays et notamment au Sud sont vastes, la superficie du seul arrondissement de Balla dans le département de Bakel et dans la région de Tambacounda, par exemple, est presque aussi importante que celle du territoire gambien voisin. De surcroît les fonds de dotations pour la décentralisation, conditionnés par un certain nombre de procédures de planification du développement, ne sont pas, en grande partie, transférés et les communautés rurales ont peu de moyens pour exercer véritablement leur pouvoir de gestion sur les ressources forestières. Aussi le recours aux aides internationales et aux projets de développement est un élément essentiel dans la mise en œuvre concrète des politiques de décentralisation.

Pourtant on ne saurait dire que le Sénégal n'est pas doté d'un ensemble de textes de lois sur la décentralisation et dans le domaine forestier suffisant pour engager des réformes concrètes dans la gestion locale des forêts. Mais, en rapprochant les lois de la décentralisation, notamment la partie relative à la gestion des forêts par les communautés rurales, et le code forestier lui même, on constate parfois certaines ambiguïtés, des points portant à controverse ou à des interprétations différentes, voire des contradictions ${ }^{5}$. Si ces ambiguïtés ne compromettent pas nécessairement le processus de décentralisation, au demeurant déjà en cours, elles le compliquent fortement et donnent lieu à des stratégies diverses de compensation, d'évitement ou de contournement des lois de la part des différents acteurs. Elles nous révèlent surtout les principaux enjeux que représentent les ressources forestières. Ceux-ci relèvent, pour le moins, de trois registres : l'accès aux ressources forestières, ou plus exactement les modes d'accès dans le sens où le pouvoir sur la gestion des ressources relève moins de l'accès direct que du mode d'accès et de son contrôle ; l'accès aux ressources financières qui sont le produit des pratiques d'exploitation légales du bois et du charbon de bois et l'accès aux ressources financières qui proviennent des activités de répression (recettes contentieuses).

L'accès aux ressources forestièresDes territoires communautaires sous conditions

Si le territoire semble rester un élément fondamental du politique, il favorise à bien des égards des stratégies multiples qui concernent, dans des formes plus ou moins autonomes ou indirectes, les modes de contrôle de l'accès aux ressources, de l'accès aux rentes et aux produits illicites, et de l'accès aux revenus des productions forestières. Ces stratégies de maintien ou de conquête du pouvoir exécutif sur la gestion des ressources forestières, passent d'abord par l'accès aux ressources.

21 En dehors des convoitises que nous avons évoquées plus haut, les forêts communautaires déléguées par la loi à la gestion des conseils ruraux, sont soumises à une condition importante d'aménagement qui limite d'emblée l'exercice du pouvoir de ces conseils. En effet, les articles R11 et R14 de la partie réglementaire du code forestier de 1998 stipulent d'une part, que "des plans d'aménagement sont requis pour toute forêt dont la superficie est supérieure à 20 hectares", et d'autre part, que pour "les forêts relevant de leurs compétences, les collectivités locales élaborent ou font élaborer des plans d'aménagement".

De la même manière toute communauté rurale doit établir un plan de gestion pour le développement local, condition pour obtenir les dotations financières prévues pour la décentralisation. Dans le domaine de la gestion des ressources naturelles, ces plans de gestion deviennent des plans d'aménagement des forêts et sont présentés ainsi comme une condition sine qua non pour accéder aux financements du Fonds forestier national. 
Du reste, les communautés rurales sont invitées à solliciter les services des eaux et forêts pour l'établissement de ces plans d'aménagement, étant entendu qu'elles n'ont pas les compétences techniques requises pour les réaliser.

Cette condition pourrait néanmoins s'envisager sous la forme d'un plan de gestion simple, dans un grand nombre de régions du Sénégal. Mais dans les régions forestières du Sud du Sénégal, c'est à dire les régions de Tambacounda et de Kolda, les deux seules ouvertes à la production de charbon de bois, cette condition devient impérative car elle est étroitement liée à la politique d'aménagement des forêts dans le cadre de l'approvisionnement urbain en combustibles ligneux (bois et charbon). Aussi les communautés rurales sont-elles contraintes de contractualiser avec les services forestiers pour réaliser des plans d'aménagement complexes dans l'objectif de produire du charbon de bois.

Une deuxième condition à la gestion des forêts par les communautés rurales est relative à la délivrance des autorisations de coupes d'arbres verts. Dans le texte de loi sur la décentralisation (art.46) c'est le président du conseil rural qui a compétence pour délivrer les autorisations préalables à toute coupe d'arbres. Dans le code forestier de 1998 (art R 20) on peut lire que "tous permis d'exploitation sont délivrés par le service des eaux et forêts". Si l'autorisation de coupe n'a qu'une valeur consultative du point de vue des forestiers, il n'en est pas de même au sein du conseil rural aux yeux duquel elle détient la même valeur exécutive que l'établissement d'un permis. Toutefois, dans la pratique, les conseils ruraux n'ont que le recours en justice, qu'ils utilisent encore très peu du fait de son éloignement et de ses contraintes, pour faire valoir leur pouvoir quand les forestiers sont, eux, assermentés pour exercer les pouvoirs policiers. Du reste, nous le verrons, à travers la mise sous aménagement des espaces forestiers communautaires, dans le cadre des projets de gestion participative, la délivrance des autorisations de coupe ne relève plus de la compétence des conseils ruraux mais des comités villageois nouvellement créés.

D'autres pouvoirs directement reliés à l'accès aux ressources, telle que la possibilité de participer à la décision d'attribution des quotas d'exploitation ${ }^{6}$ dans les forêts communautaires, restent pour les présidents de conseils ruraux des opportunités encore difficiles à saisir pour faire valoir leurs droits. Toutefois, nous avons pu assister par le passé à des oppositions de la part de quelques conseillers ruraux qui n'acceptaient plus que les productions de charbon de bois se réalisent dans leurs forêts communautaires, mais ils ont pu être contournés par des ententes établies à l'échelle de certains villages ${ }^{7}$. Là encore, l'aménagement des forêts communautaires pour la production de charbon de bois rend caduque la participation des présidents de communautés rurales aux décisions d'attribution des quotas d'exploitation dans la mesure où, au moins pour la partie aménagée, des conventions locales nouvelles contractualisent des relations directes entre les villageois et les exploitants forestiers.

Les projets de gestion participative des forestiers

Les aménagements des forêts communautaires productrices de charbon de bois dans les régions de Kolda et de Tambacounda sont l'œuvre d'un important projet de développement forestier qui a démarré en 1997 et qui a été financé jusqu'en 2004 par des crédits de la Banque Mondiale, et des dons du Royaume des Pays Bas et du gouvernement sénégalais ${ }^{8}$. Quelques forêts communautaires ont été aménagées dans les régions sud du pays, représentant 300000 ha de forêts, et concernant près de 
340 villages répartis en 7 communautés rurales. 4 de ces forêts ont vu leur plan d'aménagement finalisé en 2004.

Les opérateurs de ce projet participatif ont d'abord délimité des espaces forestiers dont ils avaient fait les inventaires des ressources ligneuses et cartographié les limites à partir de photos aériennes. Ensuite ils ont borné l'espace ciblé sur le terrain avec les villageois, puis, à l'aide de diagnostics participatifs dans les villages, ils ont invité les populations à se constituer en comités villageois de gestion. Chaque forêt peut ainsi contenir une trentaine de comités villageois de gestion.

La mise en forme de la participation, dans tous les projets de développement, se réalise selon la méthode MARP'. Celle-ci consiste en l'établissement d'un rapide diagnostic participatif de la situation du village à travers des outils élaborés afin de représenter, avec les villageois, sous forme de graphes, de cartes, de tableaux, les activités économiques, les infrastructures, les différents espaces d'habitation, de production, les espaces appropriés et communs, enfin la dynamique associative et institutionnelle du village. Il s'agit d'une incursion rapide qui rassemble tous les villageois, en général devant la concession du chef du village. Dans le même temps les animateurs du projet procèdent à "l'élection"10 des représentants du bureau du futur comité de gestion. Cette démarche intronise la plupart du temps et "tout naturellement" le chef de village comme représentant légitime, à l'occasion re-légitimé.

Les sociétés africaines sont des sociétés de palabres, mais cette parole, pour autorisée qu'elle soit à tous (exceptées les femmes si on ne les y invite pas) n'en est pas moins hiérarchiquement et très inégalement répartie (Madjarian 1991). La logique des méthodes participatives tend à nier ce contexte dès lors qu'une assemblée générale a pu rassembler la majorité des membres. Les pouvoirs des chefs de village s'en trouvent ainsi confortés, voire renforcés, et ce, dans des relations personnalisées avec les intervenants extérieurs : animateurs et responsables locaux des projets.

30 Au niveau villageois, le rôle de chaque comité est de mobiliser les résidents pour la lutte contre les feux de brousse et pour l'entretien des pare-feux (défrichements annuels et plantation d'arbres fruitiers), de surveiller les espaces forestiers contre les exploitants illicites, d'organiser et de suivre les activités annexes qui concernent les appuis au développement maraîcher, les productions apicoles, et de drainer les dons des projets (matériel, semences, vivres etc..). Dans le cadre de l'aménagement de la forêt, certains membres de ces comités ont été formés à la production de charbon de bois.

Lorsqu'ils ont tous été constitués, ces comités villageois ont été fédérés en un comité intervillageois. À cette échelle, le comité est en revanche investi d'une autre fonction, celle de gérer les ressources financières générées par la production de charbon, mais aussi par les diverses activités rémunératrices que le projet a initiées pour améliorer les revenus des populations locales. Enfin, il a également le pouvoir de délivrer les autorisations de coupe à toute personne qui en fait la demande sur le territoire concerné et se charge de relever les redevances forestières relatives à ces demandes. Bref, il est investi des pouvoirs qui reviennent, par la loi, aux conseils ruraux et il est également délégataire des tâches forestières de surveillance et de recouvrement des taxes. À travers le projet, le comité intervillageois est en relation directe avec les services forestiers envers lesquels il est redevable.

Les conseils ruraux ont-ils été oubliés? On ne saurait le dire, mais ils ont été dans une large mesure négligés, peu, voire pas du tout consultés, et ce, depuis la construction des 
premiers comités. Le contexte n'était certes pas propice à l'association des élus locaux dans la mise en œuvre du projet car celle-ci a coïncidé avec la période durant laquelle (novembre 2001- mai 2002) des délégations spéciales avaient remplacé les anciens conseillers dont les mandats avaient pris fin alors même que les élections locales n'étaient pas organisées. Si depuis lors, les conseils ruraux ont pu être à la fois informés et associés aux négociations, la logique des projets, contrainte dans des calendriers serrés et des résultats attendus, n'a cependant guère permis à certains présidents de conseil rural que de négocier a posteriori très modestement la part financière censée leur revenir dans les clés de répartition des recettes des comités Intervillageois.

L'accès aux ressources contentieuses

33 Il y a dix ans, G. Buttoud constatait que "dans la plupart des pays du Sahel [..], le produit des amendes forestières constitue la majeure partie des recettes que l'État tire de la gestion des espaces boisés. Dans cette optique, il ne s'agit pas tant de punir que de se faire de l'argent. Encore moins donc de dissuader de continuer, bien au contraire" (Buttoud, 1995 : 51). Ainsi les recettes contentieuses sont importantes, et font l'objet d'un article de loi, de la partie réglementaire du code forestier sénégalais.

Des lois et de la difficulté de les appliquer

À ce propos, le code forestier est explicite. L'article R.64 stipule :

"Les trois dixièmes du produit des amendes, confiscations, restitutions, dommagesintérêts et contraintes sont attribués aux agents des Eaux et Forêts. (...) La répartition est faite sur la base de deux dixièmes pour l'agent indicateur et $d u$ dixième pour l'agent verbalisateur. (...). Les sept dixièmes sont versés à la collectivité locale gestionnaire de la forêt dans laquelle l'infraction a été relevée ou à l'État s'il s'agit d'une infraction dans le domaine forestier de l'État".

Cet article réglementaire représente une grande avancée pour la décentralisation et la redistribution des ressources. Mais la difficulté de son application a pu être constatée à maints endroits, voire systématiquement ${ }^{11}$. Cette difficulté est majeure et elle tient au fait que l'infraction relevée dans un endroit, en l'occurrence dans une forêt communautaire, fait souvent l'objet de la production d'un acte verbalisateur dans un autre endroit, qui est en revanche déjà hors forêt communautaire. Il ne peut guère en être autrement dans la plupart des cas, puisque les actes illicites sont constatés, en général, par les villageois qui sont les plus proches des massifs forestiers étant donné que les agents forestiers n'exercent pas leur surveillance dans les massifs communautaires mais la délèguent à des équipes villageoises ou des comités de surveillance. Au demeurant, les agents forestiers sont en nombre insuffisant pour exercer leur contrôle dans les massifs qui sont sous leur responsabilité (forêts classées, zones de réserves etc.) pour être en mesure d'assurer la surveillance dans les espaces désormais dévolus aux communautés rurales.

Mais les surveillants villageois qui constatent un délit dans leur forêt, n'ont pas les moyens, ni symboliques, ni économiques, ni physiques d'exercer un quelconque pouvoir sur les contrevenants. Et c'est bien là leur drame, ou du moins celui qu'ils n'ont de cesse de présenter sous forme de doléances aux services et aux projets forestiers. "Donnez nous des bottes, des écussons, des badges, des uniformes, des armes, des bicyclettes" pour exercer la surveillance des forêts et pour être en mesure d'opposer notre autorité aux fraudeurs. Au lieu de quoi,

"Tu leur interdis, ils te disent que tu n'es rien car tu n'as pas de papier, ni de tenue, ni de bonnes chaussures et en plus, ils te menacent. On est des "forêts" que par le nom", nous précisait l'un 
de ces villageois surveillants dans une forêt communautaire de la zone périphérique du Parc du Niokolo Koba en janvier 2004". constatables sur les lieux de la communauté rurale. Et quand bien même il y en aurait, tel ce groupe de villageois qui était parvenu à arrêter un camion de chargement de charbon en situation illégale dans la forêt communautaire où il réside, les procédures de verbalisation sont longues et les taux de recouvrement des recettes contentieuses par les communautés rurales restent encore très faibles.

Les projets de surveillance participative des ressources forestières naturelles, financé par l'USAID et plus spécifiquement orienté sur le renforcement des capacités des élus locaux avait créé des équipes de surveillants communautaires en contractualisant directement avec les services forestiers régionaux des procédures selon lesquelles non seulement les $70 \%$ de recettes provenant des infractions devaient être versés dans les comptes au Trésor du budget de la communauté rural, mais en plus les $10 \%$ de l'agent indicateur devaient revenir à l'équipe villageoise de surveillance. fait, le désaccord des services forestiers régionaux, eu égard aux dispositions réglementaires $\mathrm{du}$ code forestier. D'une certaine manière le projet dépassait les prérogatives octroyées aux collectivités locales et ce, quand bien même les villageois exerçaient réellement les fonctions forestières de surveillance.

Ces exemples montrent combien sont insuffisants les mesures et les moyens envisagés. Pour autant, ils ont le mérite de poser la question de savoir qui, des villageois ou des services forestiers, dont la tâche de police forestière fait partie de leurs fonctions régaliennes, doit exercer le métier de policier.

Les codes locaux comme modes d'apprentissage de la répression

41 Nombre de projets participatifs ont investi le domaine de la gestion des ressources forestières dans des objectifs variés : production de charbon, protection de la nature, valorisation des produits forestiers non ligneux, etc. Mais ces démarches suivent souvent la même logique selon laquelle la gestion des forêts, à l'instar des politiques forestières, passe d'abord par la répression des délits. Or, "traiter la question des usages forestiers en commençant par leur faire correspondre des délits qualifiés, conduit directement à un usage répressif de la réglementation" (Buttoud, 1995 : 42). "Dans les pays où le budget de l'État est à la hauteur des revenus, c'est-à-dire des plus faibles, l'amende tend à perdre son caractère de moyen de dissuasion, pour ne devenir au contraire qu'une technique d'approvisionnement du budget, dont il n'est pas forcément espéré qu'elle ne tarisse trop vite. C'est une sorte d'impôt" (Buttoud, 1995 : 50 ).

Dans le contexte de la délimitation des espaces forestiers réalisée par les projets en vue de circonscrire qui la production de charbon, qui la surveillance des forêts, qui le contrôle des feux de brousse, les modes de contractualisation proposés aux populations villageoises tendent à enfermer ces dernières dans des espaces qui finissent par se définir en terme d'exclusion des allochtones. La définition reste élastique, bien imprécise et tend à amalgamer des acteurs aux profils bien différents: fraudeurs, délinquants, transhumants, commerçants, etc.

L'accès aux ressources financières, produits de l'exploitation du charbon de bois 
C'est ainsi que "la valorisation marchande des espaces" (Billaz et al., 2003: 178) structure de plus en plus et de manière déterminante les pratiques forestières, au-delà des réformes administratives et politiques. Bien que les conventions internationales, depuis la conférence de Rio en 1992, aient permis de mettre l'accent sur la nécessité de sauvegarder les ressources naturelles et de mettre en œuvre les programmes de lutte contre la désertification et de lutte contre la pauvreté en insistant sur l'importance des ressources forestières dans l'alimentation et le bien-être quotidien, voire en reconsidérant les valeurs sociales et symboliques des forêts, il reste que la fonction primordiale de production de bois et de charbon de bois des forêts demeure d'autant plus nécessaire que les besoins urbains sont en constante et en rapide augmentation. Depuis l'époque coloniale, "on ne s'intéresse même la plupart du temps, qu'à un seul type de produit, c'est-à-dire le bois dont les caractéristiques correspondent aux utilisations commerciales ou industrielles. C'est celui-ci qui paie une redevance d'exploitation, et qui rapporte donc à l'État" (Buttoud op. cit : 41).

En l'occurrence, au Sénégal, nous insisterons davantage sur l'utilisation commerciale du bois dans le but de produire le charbon de bois.

Des lois non suivies d'effets

Dans le code forestier de 1998, reformulé afin de s'adapter aux nouvelles lois de la décentralisation, il est clairement stipulé que les mécanismes d'attribution des quotas d'exploitation pour les exploitants forestiers producteurs de charbon de bois, disparaîtraient en l'espace de trois ans, c'est-à-dire le 21 février 2001 (article R.66).

"Nonobstant cette disposition réglementaire pourtant non équivoque, on remarque une survivance du système du quota dans la pratique renforcée par d'autres dispositions réglementaires ${ }^{12}$. Mais juridiquement, les arrêtés ministériels organisant les campagnes forestières depuis 2001 violent les dispositions du code forestier"13.

Des projets participatifs au bénéfice de qui ?

Le projet de gestion participative des forestiers évoqué plus avant avait pour principal objectif de former les villageois riverains de l'espace forestier mis sous aménagement à la production de charbon de bois. La constitution des comités de gestion villageois devait servir, à terme, aux villageois formés à la production de charbon de base pour se structurer ensuite en Groupement d'Intérêt Économique (GIE), seule instance juridique reconnue par l'État pour obtenir les patentes d'exploitant forestier, et intégrer ainsi la filière économique si fortement monopolisée.

En fait, les linéaments du projet, étroitement liés à la politique forestière, n'ont pas permis aux villageois nouvellement formés d'entrer dans la filière en concurrence avec les patrons charbonniers. Non seulement les patentes ne leur ont pas été attribuées, mais ils n'ont pas non plus bénéficié des appuis nécessaires (crédits, soutiens administratifs) pour maintenir la concurrence. Le projet lui-même est en panne et une partie des bailleurs s'est retirée. L'argument selon lequel les villageois formés sont trop peu nombreux pour couvrir la demande urbaine de charbon de bois revêt, de fait, un caractère tautologique.

8 Les productions de charbon de bois déterminent les politiques forestières qui ont, par conséquent, du mal à prendre en compte les processus de décentralisation. Et il n'est pas tant question ici de la seule difficulté de reformer le service forestier dans ses fonctions régaliennes de contrôle d'un territoire ou de police environnementale, que du poids économique laissé à un puissant oligopole : celui des patrons charbonniers. 

pouvoir ait été centralisé. Or, ces auteurs constatent que dans nombre de pays africains, le pouvoir est davantage concentré entre les mains d'une personne, d'un parti, d'un clan ou d'un réseau de clientèle ${ }^{14}$. Dans ces cas là, suggère-t-il, la solution se trouverait davantage dans une déconcentration plutôt que dans une politique de décentralisation. Cette dernière, dans ce contexte, ne permettrait pas "d'espérer un bénéfice quelconque (...) par la privatisation ou par une redistribution des pouvoirs" (Husson, 1997 : 32).

Les pouvoirs décentralisés et les pouvoirs déconcentrés ne sont pas des vases communicants. Des élus responsables Tambacounda et de Kolda au Sénégal, au pourrait croire que les élus des conseils ruraux sont immobiles, paralysés et dans l'incapacité d'agir face à des pouvoirs d'État trop concentrés. Pourtant les exemples ne manquent pas pour décrire les actes posés par des présidents de $\mathrm{CR}$ pour faire valoir leurs droits en matière de gestion des ressources forestières. Certains ont pu faire acte de résistance face aux demandes d'exploitation du charbon de bois, d'autres ont pu s'opposer également, à travers les commissions domaniales, aux demandes multiples de privatisation des espaces communautaires de la part des politiques, de la part des chefs religieux ${ }^{15}$ et / ou d'entrepreneurs économiques.

51 Les élus sont plus nombreux aujourd'hui qu'il y a seulement 4 ans à connaitre les lois forestières. Ainsi, si en 2001, seulement $28,2 \%$ des collectivités locales connaissaient la loi sur la répartition des recettes contentieuses ${ }^{16}$, nous avons pu identifier une proportion double en 2004, sur un échantillon restreint de 5 communautés rurales de la région de production de charbon de bois ${ }^{17}$. Enfin, bien que soumis encore dans la majorité des cas à la justice des inspections forestières, nombre de litiges au sujet des recettes contentieuses sont en cours.

52 Avec de nombreux appuis en formation, les élus locaux répondent mieux de leurs actes, participent aux initiatives de certains médias qui mobilisent les citoyens à la base, soit à travers des informations sur leurs droits et devoirs, soit à travers des débats hebdomadaires animés au cours desquels les élus répondent à leurs administrés. Ces "geewu askanwi", (assemblée populaire) telle que celle mise en place par une radio locale à Fissel, sont des expériences intéressantes de participation à la gestion des affaires publiques ${ }^{18}$.

53 Cependant, bien des limites existent encore. Ces espaces pour la participation délibérative sont en nombre réduit, de facture plus urbaine que rurale. Les mécanismes de contrôle des élus par les citoyens restent en deçà des attentes de la décentralisation. Les Conseils ruraux sont aussi vus comme le lieu de conflits politiques et de factionnalisme (Blundo, 1996:119), de rapports clientélistes pour l'accès aux ressources, encouragés en partie par le jeu concurrentiel que se livrent certains projets de développement sur les mêmes territoires.

L'État domine mais ne contrôle pas

54

Quand bien même une certaine vision libérale présente les politiques de décentralisation comme alternatives aux modes de gouvernement trop centralisé, la décentralisation et la déconcentration ne sont pas des phénomènes de même nature et ne sont pas opposés. Cependant, la politique de déconcentration des services de l'État n'est pas, loin s'en faut, une volonté politique affichée avec le même enthousiasme que 
celle de la décentralisation. On pouvait lire en 2003, dans le document du Plan d'action pour la Décentralisation, qu'un projet de loi portant charte de la déconcentration et les projets de décrets respectivement relatifs à l'organisation de l'administration centrale et instituant un comité interministériel de la déconcentration datent de novembre 1998 et n'ont pas été suivis d'effet ${ }^{19}$.

La légitimité de l'État passe pourtant par un équilibre de sa stratégie globale d'intervention avec les projets décentralisés, par l'amélioration de la déconcentration des fonctions étatiques territoriales et par l'approfondissement des moyens de contrôle a posteriori de la légalité des actes des collectivités locales et des budgets.

Ces éléments minimaux nécessaires à l'exercice légitime d'un service déconcentré de l'État ne sont pas exhaustifs mais ils montrent déjà l'ampleur du déséquilibre entre la décentralisation et la déconcentration. En définitive, il n'existe pas, comme le laisse supposer le plan d'action de la décentralisation, de "culture" de la déconcentration auprès des autorités centrales, pas plus qu'il n'existe de "noblesse législative" pour cette déconcentration, comme il en existe une pour la décentralisation ${ }^{20}$.

Conclusion

Si le processus de décentralisation au Sénégal a une histoire ancienne et bien peu linéaire, l'exemple de la gestion des ressources forestières nous donne à voir qu'il est également un processus dont on pourrait croire qu'il est un peu compromis, mais qui apparaît aussi comme un peu compromettant. À travers ses limites, il donne à voir le rôle structurant du marché.

Les projets participatifs de gestion des ressources forestières tendent à favoriser un certain statu quo. Conduits par les forestiers eux-mêmes, dans le cadre des aménagements forestiers et des politiques énergétiques d'approvisionnement des villes en combustibles ligneux, ils font des forêts communautaires des espaces en partie soustraits à l'autorité des conseils ruraux. Les modes de régulation et de gestion des ressources que procurent ces forêts sont directement contractualisés avec les chefs de village, dans des relations parfois personnalisées, laissant à la marge les élus locaux aussi bien dans l'exercice de leur pouvoir que dans la redistribution des recettes (de la production et de la répression).

L'apprentissage de la démocratie demeure problématique et les relations hiérarchiques sont renforcées défavorisant l'apprentissage de la construction d'un espace citoyen au sein des collectivités locales. Quand bien même certains projets, comme celui de l'USAID, ont contribué à renforcer les capacités d'un certain nombre de conseils ruraux de la région, et ont permis d'envisager le recouvrement des amendes que les textes de lois prévoient en faveur des communautés rurales, leurs démarches participatives n'ont cependant pas permis de rendre autonomes des comités villageois dans la surveillance des forêts. Mais était-ce bien là leur rôle?

Dans leur existence éphémère, ils laissent en suspens des formes de mobilisation des populations qui, à l'instar des politiques forestières, privilégient les modes de répression comme source de revenus. Le mouvement de la décentralisation au Sénégal, en matière de gestion forestière, ne desserre pas l'emprise du service forestier sur le contrôle des forêts, mais semble, dans une vision post-moderne ${ }^{21}$, écarteler le Sénégal entre une logique du marché et un repli communautaire. 


\section{BIBLIOGRAPHIE}

Billaz R., 2003, "Les États francophones d'Afrique de l'Ouest face à la décentralisation, le poids et les acquis du passé . Et si l'on interrogeait l'histoire coloniale ?", in Totté M., Dahou T. et Billaz R. (ss-dir.), La décentralisation en Afrique de l'Ouest. Entre politique et développement, Bruxelles, Paris, Dakar, Cota- Karthala- Enda Graf.

Blundo G., 1996, "Gérer les conflits fonciers au Sénégal : Le rôle de l'administration locale dans le sud-est du bassin arachidier", in Tersiguel, P. et Becker.C., (eds.), Développement durable au Sahel, Karthala, Paris, Dakar.

Boutinot L., 2002, "De la complexité de la décentralisation. Exemple de la gestion des ressources forestières au Sénégal", Bulletin de l'APAD, 23, septembre 2002.

Buttoud G., 1995, La forêt et l'État en Afrique sèche et à Madagascar. Changer de politiques forestières, Paris, Karthala.

Cabinet CIG, 2001, Bilan de la décentralisation., Dakar : USAID - Ex- MATD, disponible sur le site www.CIG.SN/CNCR

Dieng C., Diaham B., 1999, Diagnostic de la filière des produits forestiers de cueillette et perspectives de développement, Dakar MENPN/ DEFCCS/ Progede.

Diop M.C., (ss-dir.) 2004, Gouverner le Sénégal. Entre ajustement structurel et développement durable, Paris, Karthala.

Diop M.C., 2004, "Essai sur "l’art de gouverner" le Sénégal", in : Diop, M.C. (ss-dir.), Gouverner le Sénégal. Entre ajustement structurel et développement durable, Paris, Karthala.

Gueye B., 1998, Les approches participatives, IIED, Dakar.

Husson B., 1997, "La problématique en débats : entre indigénités et modernités", in GEMDEV (ed.), Les avatars de l'État en Afrique, Paris, Karthala.

Lavigne-Delville P., Sellamna N-E., Mathieu M., (ss-dir.) 2000, Les enquêtes participatives en débat. Ambition, pratiques et enjeux, Paris, GRET- Karthala- ICRA.

Madjarian G., 1991, L'invention de la propriété. De la terre sacrée à société marchande, Paris, L'Harmattan.

Olivier de Sardan J-P., 2004, "Des pouvoirs locaux dans l'attente de la décentralisation au Niger", Études et Travaux, 27, Lasdel, Niamey.

Ribot J.C., 1990, "Politique forestière et filière du charbon de bois", in : Bergeret, A., L'arbre nourricier en Afrique sahélienne, Paris, Maison des Sciences de l'Homme.

Ribot J., 1995, "Le contrôle local des forêts au Sénégal : vers des politiques participatives", Center for Population and Development Studies, Harvard University.

Samb M., 2004, "La gouvernance publique : changement ou continuité ?", in Diop, M.C., (ss-dir.), Gouverner le Sénégal. Entre ajustement structurel et développement durable, Paris, Karthala.

Thiam B., "Décentralisation au Sénégal : les bœufs attendent la charrue", Panos Info, 15, sd.

Thiaw S., Ribot JC., 2003, "Privileging Outsiders, Excluding Insiders: Forest Access through Village Chiefs in Senegal", Paper prepared for : The International Conference on Competing Jurisdictions : Settling Land Claims in Africa, Vrije Universiteit Amsterdam, 24 - 27 September 2003. 
Weber J., 2000, "Pour une gestion sociale des ressources naturelles", in Compagnon, D. et Constantin, F., 2000, Administrer l'environnement en Afrique, Paris- Nairobi Karthala - IFRA.

Werner J.-F., 1997, "Itinéraires individuels à la marge. Études de cas sénégalais", in Marie, A. (ed.), L'Afrique des individus, Paris, Karthala : 367-403.

Code Forestier, 1965, Journal Officiel. 3730 du 6 mars 1965.

Code Forestier, 1972, Journal Officiel : 1351, Décret N 73-1351 du 17 novembre 1972.

Code forestier, 1998, Loi 98-03 du 8 janvier 1998, Décret Nº 98-164 du 20 février 1998.

Décret $\mathrm{N}^{\circ}$ 65-078 du 10 février 1965 (partie réglementaire), Loi $\mathrm{N}^{\circ}$ 65-23 du 9 février 1965 (partie législative).

Loi 74-46 du 28 Juillet 1974, Journal Officiel N 4375 du 28 juillet 1974 (partie législative).

Loi 93-06 du 4 février 1993, Décret 95-357 du 11 avril 1993.

Textes de lois et des décrets sur la décentralisation du 27 décembre 1996.

\section{NOTES}

1. Cf. les travaux en cours que je coordonne dans le cadre d'un programme financé par le CIRAD sur la comparaison des processus de transfert de gestion des ressources naturelles au Mali, au Niger et au Sénégal, ainsi que ceux de l'observatoire de la décentralisation du LASDEL à Niamey coordonné par J.-P. Olivier de Sardan.

2. La DEFCCS est la Direction des Eaux et Forêts, de la Chasse et de la Conservation des Sols, sous la tutelle du Ministère de l'Environnement et de la Protection de la Nature (MEPN).

3. Je renvoie le lecteur à mon article cité. Boutinot L., 2002.

4. Entretien avec un inspecteur forestier régional.

5. Cf Boutinot L., 2002.

6. Les quotas d'exploitation sont des quantités de stères de bois ou de kilos de charbon de bois autorisés à la vente quels que soient les lieux de coupe et les surfaces d'exploitation nécessaires pour les obtenir. Ces quotas sont distribués à des coopératives agréées dont quelques unes seulement dominent le marché constituant un véritable oligopole (cf : J. Ribot, 1990, 1995).

7. Thiaw S. et Ribot J., 2003.

8. État sénégalais : 1,2 Mds de \$ et Banque Mondiale et Pays Bas : 19,9 Mds de \$.

9. Méthode accélérée de Recherche Participative, devenue Méthode Active de Recherche et de Planification Participative. Pour une analyse fine et une critique constructive des enquêtes participatives, je renvoie le lecteur à l'important ouvrage de Philippe Lavigne-Delville, Nour Eddine Sellamna, et Marie Lou Mathieu (ss-dir.), 2000.

10. Autrement dit à la désignation à main levée ou par consensus.

11. Cf les travaux de C. N. Diouf sur "la gestion participative dans la forêt de Saré Gardi", document de travail décembre, 2004, Programme ATP Cirad. De même ceux de Thiaw, Sagane, op.cit. et de Papa Faye, "Le pluralisme institutionnel dans la gestion décentralisée des ressources naturelles", document de travail, 2004, Prog. WRI-CIRADCODESRIA.

12. L'arrêté portant organisation de la campagne 2004 donne une base légale au quota actuel ; c'est cet arrêté qui fixe lui-même la répartition du quota. 
13. Cf. BA El Hadj Djaligué, La réglementation de la filière du charbon de bois à l'épreuve de la décentralisation : entre discours, lois et pratiques, Document de travail, Programme WRI-CIRA- CODESRIA, "Pour une décentralisation démocratique de la gestion des ressources forestières au Sénégal", Dakar, mars 2005.

14. Ces propos de E Le Roy sont cités par M.C.Diop, 2004, op.cit p 20. Cf également Husson B., 1997 : 32.

15. Au cours d'une délibération d'un conseil rural de la région de Kolda, à laquelle nous assistions en 2004, nous avons pu observer l'usage du registre religieux sur lequel s'appuient avec insistance les entrepreneurs privés pour demander des terres, après que leurs stratégies de contournement du Conseil rural ont échouées.

16. "Avec seulement $36,4 \%$ de ces recettes qui sont recouvrées pour celles-ci", Cabinet CIG, 2001, Bilan de la Décentralisation, p 47.

17. Cf. Thiaw, Sagane, Les conseils ruraux dans la décentralisation de la gestion des forêts. Document de travail, Programme WRI-CIRAD-CODESRIA, février 2005.

18. Thiam B., op.cit.

19. Plan d'action de la décentralisation 2003-2005, Ministère de l'Intérieur, Ministre délégué chargé des collectivités locales, 21 février 2003. p 41.

20. Op.cit., p 34-35.

21. Pour reprendre l'image de J.F. Werner, 1997.

\section{AUTEUR}

\section{LAURENCE BOUTINOT}

CIRAD, Dakar, Sénégal 